\title{
La respuesta de modelos espectrales de olas en condiciones de huracanes intensos en el golfo de México
}

\section{Response of spectral wave models under extreme wind conditions in the Gulf of Mexico}

\author{
Rodney Eduardo Mora Escalante ${ }^{1,2,3^{*}}$ \& Omar Gerardo Lizano Rodríguez ${ }^{1,3}$
}

\begin{abstract}
RESUMEN
Se evaluó la respuesta de dos modelos numéricos de oleaje de tercera generación en condiciones de viento extremo, un modelo de oleaje de aguas profundas, WAVEWATCH III y un modelo de olas de aguas someras, SWAN. El objetivo general del trabajo fue evaluar el desempeño de dos modelos espectrales de olas para representar las condiciones de altura de oleaje de un huracán con viento generado por dos tipos de modelos de viento: uno paramétrico y otro dinámico. Se realizó 4 experimentos con huracanes mayores e históricos que atravesaron el golfo de México (Lili 2002, Iván 2004, Rita 2005 y Gustav 2008). Para comparar los resultados de los modelos se utilizó datos observados de rapidez del viento y altura de oleaje de boyas de la National Data Buoy Center y se evalúa con parámetros estadísticos. Los resultados mostraron que al usar el campo de viento del modelo paramétrico en los modelos SWAN y WAVEWATCH III, ambos tienden a representar los máximos de altura significante del oleaje con lo observado, cuando la trayectoria de la tormenta pasa cerca de la boya de registro $(<400 \mathrm{~km})$. El modelo WAVEWATCH III forzado con el campo de viento del modelo WRF tiende a subestimar el máximo de altura de oleaje observado. La estadística evaluada y las series temporales de rapidez de viento y altura de oleaje, mostró que el modelo SWAN y el modelo WAVEWATCH III forzados con el modelo paramétrico HURWIN son adecuados en la simulación de oleaje en condiciones de huracanes mayores.
\end{abstract}

Palabras clave: altura significante del oleaje, golfo de México, huracán, SWAN, WAVEWATCH III

\begin{abstract}
Two numerical wave models were evaluated in extreme wind conditions: a deep-water wave model, WAVEWATCH III, and a shallow water wave model, SWAN. The general objective of the research is to assess the performance of these two spectral wave models to represent the wave height under hurricane conditions with two kinds of wind models: parametric and dynamic. Four experiments were conducted with major historical hurricanes that crossed the Gulf of Mexico

1 Centro de Investigación en Ciencias del Mar y Limnología (CIMAR), Ciudad de la Investigación, Universidad de Costa Rica, Montes de Oca, San Pedro, 11501-2060 San José, Costa Rica. rodney.moraescalante@ucr.ac.cr* ORCID: http:// orcid.org/0000-0001-7484-8299, omarglizano@gmail.com ORCID: https://orcid.org/0000-0001-9407-0322

2 Escuela de Física, Sede Rodrigo Facio, Universidad de Costa Rica, San Pedro,11501-2060 San José, Costa Rica

3 Módulo de Información Oceanográfica (MIO), Centro de Investigación en Ciencias del Mar y Limnología, Universidad de Costa Rica. Ciudad de la Investigación, Finca 2, Edificio CIMAR
\end{abstract}


(Lili 2002, Ivan 2004, Rita 2005, and Gustav 2008). In order to compare results from the models, in-situ data of wind speed and significant wave height was obtained from the National Data Buoy Center and evaluated using statistical parameters. Results showed that, by using the parametric wind field model in SWAN and WAVEWATCH III, both models tend to represent the peaks of observed significant wave height when the storm path passes close to the measuring buoy ( $<$ $400 \mathrm{~km}$ ). Forced by the wind field of the WRF model, WAVEWATCH III tends to underestimate the peak wave height. The evaluated statistics and the time series of wind speed and wave height show that, forced by the HURWIN parametric model, SWAN and WAVEWATCH III are adequate for wave simulation under major hurricane conditions.

Keywords: significant wave height, Gulf of Mexico, hurricane, SWAN, WAVEWATCH III

\section{INTRODUCCIÓN}

Existen una serie de modelos que simulan el viento para un huracán, tal como los modelos paramétricos de viento que utilizan relaciones muy sencillas (Holland, 1980; Lizano, 1990; Willoughby \& Rahn, 2004; Holland et al. 2010; Emanuel \& Rotunno, 2011; Hu et al. 2012); también están aquellos que utilizan ecuaciones más complejas para representar la dinámica de la atmósfera como el modelo Weather Research and Forecasting, WRF (Skamarock et al. 2008). Estudios recientes emplean la técnica de combinar información de viento con datos de reanálisis y modelos paramétricos, para simular el campo de viento en condiciones de huracán (Montoya et al. 2013; Shao et al. 2018; Chen et al. 2019).

El empleo de modelos de mesoescala (por ejemplo, el WRF) en la simulación de huracanes para evaluar su física (capa límite planetaria, cúmulos, microfísica, entre otros) y parámetros de pronóstico como intensidad, estructura y trayectoria de la tormenta, han sido usados con éxito (Braun \& Tao, 2000; Rogers et al. 2003; Davis et al. 2008; Mooney et al. 2016). También se han implementado modelos paramétricos para la simulación de viento de huracanes: como en el caso del ciclón tropical Joan de 1988 (Lizano \& Moya, 1990; Lizano et al. 2001; Ortiz-Royero et al. 2008) y Mitch 1998 (Lizano et al. 2001) por su paso por el Caribe y Centroamérica. En el estudio previo de Olfateh et al. (2017), se utilizó un nuevo modelo paramétrico que usa 6 huracanes en la región del Atlántico Norte para evaluar el modelo de viento. El modelo propuesto demuestra capturar los principales rasgos de la tormenta (asimetría, viento máximo, y otros). En el trabajo reciente de Yaghoobi Kalourazi et al. (2020) se evalúa una serie modelos paramétricos usando datos de vientos de $\mathrm{H}^{*}$ Wind, de reanálisis y de boyas en el golfo de México, al demostrar que los modelos analíticos que generan el campo de viento en huracanes son consistentes con las observaciones. 
En los modelos numéricos de oleaje, la principal fuente de energía en la generación del oleaje es el viento o es el esfuerzo de este (Holthuijsen, 2010). Estos modelos están basados en la ecuación de balance de acción de la ola, $\mathrm{DN}(\mathrm{x}, \mathrm{y}, \mathrm{t} ; f, \theta) / \mathrm{Dt}=$ $\mathrm{S}$ (Komen et al. 1994). El término de la izquierda es la derivada total que indica el cambio local y propagación de la acción de la ola $(\mathrm{N}=\mathrm{E}(f, \theta) / \theta$; $\theta=2 \pi f$; donde $\mathrm{E}, \mathrm{f}, \theta$ es la energía del espectro, frecuencia, dirección de las olas, respectivamente, y $\theta$ es frecuencia angular). El lado derecho controla el crecimiento/decrecimiento del oleaje definido por los términos fuente y de sumidero $(\mathrm{S}=$ Energía del viento + Intercambio de energía entre olas + Disipación de energía por rompimiento de las olas; válido para aguas profundas). En Lizano (2006), al emplear el modelo paramétrico ajustado de viento de huracán (Lizano, 1990) a un modelo de oleaje de tercera generación, SWAN, los perfiles de altura de oleaje generados por el modelo numérico en 5 huracanes simulados, dio un mejor ajuste que el modelo de Holland (1980) de viento. Los resultados en dicho estudio demuestran que el empleo de ambos modelos pueden ser una técnica útil para diagnosticar y pronosticar el oleaje durante ciclones tropicales. Otros autores como Moon et al. (2003) mencionan que la simulación de oleaje con un modelo numérico de tercera generación como WAVEWATCH III y forzado con el campo de vientos de un modelo paramétrico, produjo una buena consistencia entre los resultados del modelo y los datos observados en boyas marinas. En un estudio reciente de Ruiz-Salcines et al. (2019) se evalúa el campo de viento al usar 6 diferentes modelos paramétricos para huracanes y la respuesta del campo de olas que utiliza el modelo MIKE21. Uno de los hallazgos que se mencionan es, los modelos paramétricos pueden ser precisos para algunos eventos, pero para otros pueden encontrarse imprecisiones en los resultados. Además, los resultados del campo de viento y de olas son dependientes de la distancia al centro del huracán. En otro estudio, Lizano \& Mora-Escalante (2019) muestran que los resultados de altura y periodo del oleaje del modelo SWAN forzado con el campo de vientos de un modelo paramétrico son similares a las mediciones de una boya ubicada cerca de la costa caribe de Costa Rica.

Luego de varias décadas, se siguen evaluando una serie de modelos paramétricos de viento de huracán que existen en la literatura, que, por su simplicidad, son usados en la simulación de oleaje extremo. Este estudio retoma el modelo paramétrico de viento de Lizano (1990) para evaluar la respuesta de 2 modelos numéricos de oleaje (SWAN y WAVEWATCH III) en representar la altura de oleaje 
en condiciones de huracán en la región del golfo de México; además se evalúa la respuesta de estos dos modelos de oleaje con otro campo de viento generado con un modelo más complejo.

\section{MATERIALES Y MÉTODOS}

El área de estudio comprende el golfo de México, Cuba y el norte de México, es decir, $18^{\circ} \mathrm{N}-31^{\circ} \mathrm{N}$ y $80^{\circ} \mathrm{O}-95^{\circ} \mathrm{O}$ (Fig. 1). Además, en la Fig. 1 se indican las trayectorias de los ciclones tropicales Lili 2002, Iván 2004, Rita 2005 y Gustav 2008 que se incluyeron para el análisis; así como las ubicaciones de cinco boyas oceanográficas administradas por el Centro Nacional de Datos de Boyas (National Data Buoy Center, NDBC) utilizadas en el estudio. Las boyas 42001 (3 207 m) y 42003 (3 $296 \mathrm{~m}$ ) están en aguas profundas entre tanto, las boyas 42 $036(49.7 \mathrm{~m}), 42039$ (270 m) y 42040 $(183 \mathrm{~m})$ se encuentran en aguas intermedias o someras.

Para los 4 huracanes se usó un modelo de viento paramétrico y un modelo dinámico para obtener el campo de viento a $10 \mathrm{~m}$ sobre la superficie en el dominio mostrado en la Fig. 1. Estas tormentas se seleccionaron por categorizarse como huracanes mayores, porque durante el mayor tiempo de su trayectoria por aguas del golfo de México alcanzaron categoría $\geq 3$ en la escala
Saffir-Simpson de acuerdo con la intensidad de sus vientos.

Para la simulación del campo de vientos de un huracán se emplearon dos modelos de viento que se diferencian en la forma de calcular la magnitud del viento. Uno de los modelos que se implementa es el modelo ajustado de Lizano (1990) (de aquí en adelante referido como HURWIN). El perfil de viento de este modelo consiste en una combinación de los modelos de Jelesnianski (1974): $U_{\text {máx }}\left[\frac{2 R_{\text {máx }} r}{R_{\text {máx }}^{2}+r^{2}}\right]$ para $\mathrm{r}<\mathrm{R}_{\text {máx }}$ y Collins Viehnaman (1971): $U(r)=\frac{U_{\text {máx }}}{C_{1} r^{k}} \log \left[\frac{R_{\text {máx }}}{C_{2} r^{m}}\right]$ para $\mathrm{r}>\mathrm{R}_{\text {máx }}$, donde $\mathrm{C}_{1}=3.354, \mathrm{C}_{2}=1.265 \times 10^{-3}, \mathrm{k}$ $=0.15128 \mathrm{y} \mathrm{m}=1.607$. El viento máximo se calcula con la siguiente expresión, $U_{\text {máx }}=0.885\left(10.8866 \sqrt{P-P_{c}}-f R_{\text {máx }}\right)+0.5 V_{t}$ con $f$ parámetro de Coriolis, $\mathrm{P}$ presión ambiental (1016 mb), Pc presión mínima central y $\mathrm{V}_{\mathrm{t}}$ es la velocidad de traslación. De los parámetros más difíciles de obtener es el radio de viento máximo $\left(\mathrm{R}_{\text {máx}}\right.$, por lo tanto, en este estudio se adopta la expresión sugerida por Vickery \& Wadhera (2008), válida para el golfo de México, $\ln \left(R_{\text {máx }}\right)=3.858-7.70 \times 10^{-5}(\Delta p)^{2}$, donde $\mathrm{R}_{\text {máx }}$ es el radio de viento máximo, $\Delta p=P-P_{c}$. Los parámetros de entrada en el modelo paramétrico son la posición (latitud y longitud) del centro de la tormenta y presión mínima central. Se obtienen de la base de datos HURDAT2 (Landsea \& Franklin 2013) del 
Centro Nacional de Huracanes (NHC, por sus siglas en inglés). Los campos de viento se interpolan a una altura de 10 $\mathrm{m}$ sobre el nivel del mar y con una resolución temporal y espacial de 3 horas y $\sim 10 \mathrm{~km}$, respectivamente.

El otro modelo de viento que se utilizó en el estudio es el dinámico no hidrostático Advanced Research WRF (Skamarock et al. 2008) (núcleo dinámico ARW) versión 3.0. Se configura con 3 dominios anidados con resolución espacial de $90 \mathrm{~km}, 30$ $\mathrm{km}$ y $10 \mathrm{~km}$ y resolución temporal de 3 horas. Se emplean las formulaciones físicas por defecto para representar los diferentes procesos en la atmósfera. Para el estudio se utiliza el dominio más fino. Se usa información extraída del reanálisis de la National Center for Environmental Prediction (NCEP) Final Analysis (FNL) como condiciones iniciales y de frontera en el modelo de mesoescala. Estos datos tienen una resolución espacial de $1^{\circ}$ y un intervalo temporal de 6 horas. Cabe indicar, que se simuló para cada tormenta una única vez el campo de viento.

Para simular el campo de oleaje se emplearon 2 modelos numéricos de oleaje, SWAN y WAVEWATCH III (de aquí en adelante referido como WW3). Para el modelo numérico WW3 se utiliza la versión 4.18 (Tolman, 2014). Para el modelo espectral SWAN (Simulating Waves Nearshore) se usa la versión 41.10
(Booij et al. 1999). La batimetría que se emplea para propagar el oleaje de los 2 modelos es ETOPO1 (Amante \& Eakins, 2009). La resolución espacial en los 2 modelos de oleaje es la de ETOPO1. El rango de frecuencias del oleaje es de $0.04-0.4 \mathrm{~Hz}$ (incremento en frecuencia de forma logarítmica, $1.1 \mathrm{~Hz}$ ) y 36 direcciones para definir el espectro de energía de las olas $\left(\Delta \theta=10^{\circ}\right)$. Los términos fuente y sumidero utilizados fueron la de Tolman \& Chalikov (1996) en WW3 (se establece un valor de coeficiente de arrastre limitado de $3 \times 10^{-5}$ en el término de crecimiento de la ola), mientras que la física por defecto se emplea en el modelo SWAN. El método aproximado DIA fue empleado en los 2 modelos numéricos para resolver las interacciones no lineales entre olas. Para mayores detalles en la física de ambas parametrizaciones de los modelos de oleaje WW3 y SWAN refiérase a Ortiz-Royero \& Mercado-Irizarry (2008).

Se usó resultados de rapidez de viento y de altura significante del oleaje (cantidad obtenida como un parámetro integral del espectro de energía del oleaje) de los modelos cada hora e interpolado en las posiciones mostradas en la Fig. 1. Para comparar los resultados del modelo de viento y de oleaje, se utilizó datos horarios de observaciones de boyas oceanográficas en el golfo de México (Fig. 1). Para evaluar el desempeño de los 2 modelos de viento 
en los 2 modelos de oleaje empleados en las diferentes simulaciones realizadas se aplican las siguientes métricas estadísticas definidas en Schiller \& Brassington (2011): el error medio, $M E=\bar{M}-\bar{O}$ el error medio cuadrático, $R M S E=\sqrt{\frac{1}{N} \sum_{i=1}^{N}\left(M_{i}-O_{i}\right)^{2}}$ y el coeficiente de correlación,
$R=\frac{\sum_{i=1}^{N}\left(M_{i}-\bar{M}\right)\left(O_{i}-\bar{O}\right)}{\sqrt{\sum_{i=1}^{N}\left(M_{i}-\bar{M}\right)^{2}} \sqrt{\sum_{i=1}^{N}\left(O_{i}-\bar{O}\right)^{2}}}$. La M representa el modelo y la $\mathrm{O}$ indica las observaciones. La barra sobre las letras $\mathrm{M}$ y $\mathrm{O}$ denota el promedio y la $\mathrm{N}$ es el número de datos.

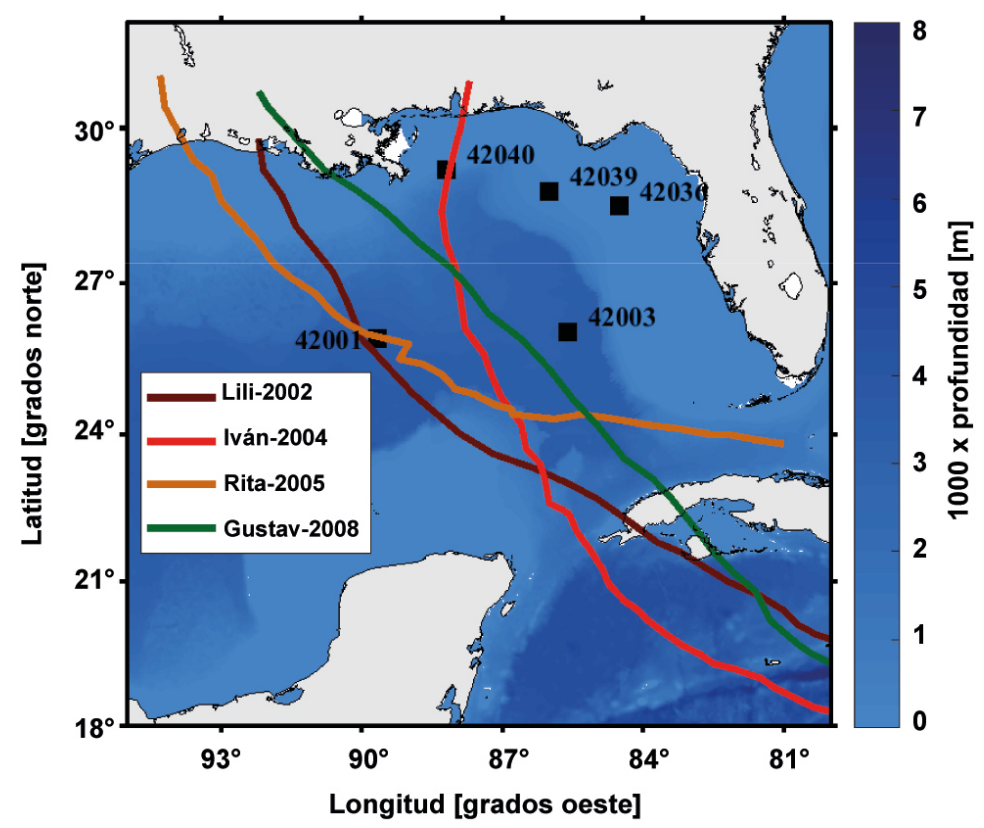

Fig. 1. Región de estudio que abarca el golfo de México. Los cuadros negros indican boyas marinas de la NDBC. Los tonos de azul la batimetría. Las líneas de colores representan la trayectoria de los ciclones tropicales de acuerdo con la información del NHC

Fig. 1. Study region covering the Gulf of Mexico. Black squares represent NDBC buoys, blue shades indicate bathymetry, and color lines denote the trajectory of tropical cyclones based on NHC information 


\section{RESULTADOS}

Para comparar los resultados de los modelos de viento HURWIN y WRF, se presentan series temporales de la magnitud del viento según la posición de la boya con respecto a la trayectoria de la tormenta. También se muestran los resultados de las series temporales de la altura significante del oleaje con el modelo WW3 y el SWAN, según la ubicación de la boya en aguas profundas y aguas intermedias o someras. Finalmente se evalúa la respuesta de los dos modelos de oleaje ante los dos modelos de viento empleados en el estudio con criterios estadísticos.

Comparación de la rapidez del viento en boyas lejanas a la trayectoria de la tormenta

Los resultados de la rapidez del viento para los 4 huracanes simulados con los dos modelos de viento se muestran en la Fig. 2 y Fig. 3, y en las posiciones de las boyas (42 036, 42 039 y 42 040) que están lejos de la trayectoria de la tormenta. En la Fig. 2 se muestra la serie temporal para los huracanes Iván (Fig. 2A, C) y Gustav (Fig. 2B, D). En ninguna de las boyas se registraron valores de intensidad del viento mayores a $30 \mathrm{~m} \mathrm{~s}^{-1}$, lo que reafirma la escogencia de estas boyas como distantes a la influencia de estas tormentas. En general los resultados de rapidez de viento con el modelo WRF (línea azul) tienden a ser consistentes con las observaciones registradas en las boyas 42036 y 42 039, mientras que el HURWIN (línea roja) es pobre en representar estas condiciones.

La Fig. 3 muestra la serie temporal para el huracán Lili (lado izquierdo) y para el Rita (lado derecho). Igual que en la Fig. 2, los resultados de la magnitud del viento con el WRF (línea azul) tienden a ser similares con los datos medidos en las boyas 42039 (Figs. 3A, C) y 42040 (Figs. 3B, D); además la intensidad del viento en los registros de ambas boyas de los 2 huracanes no supera los $20 \mathrm{~m} \mathrm{~s}^{-1}$. Es notorio de las Figs. 2 y 3 que el campo de vientos generado por HURWIN no es adecuado para representar el viento muy lejos del centro de la tormenta, mientras que el modelo WRF representa bien las condiciones ambientales de viento moderado. 

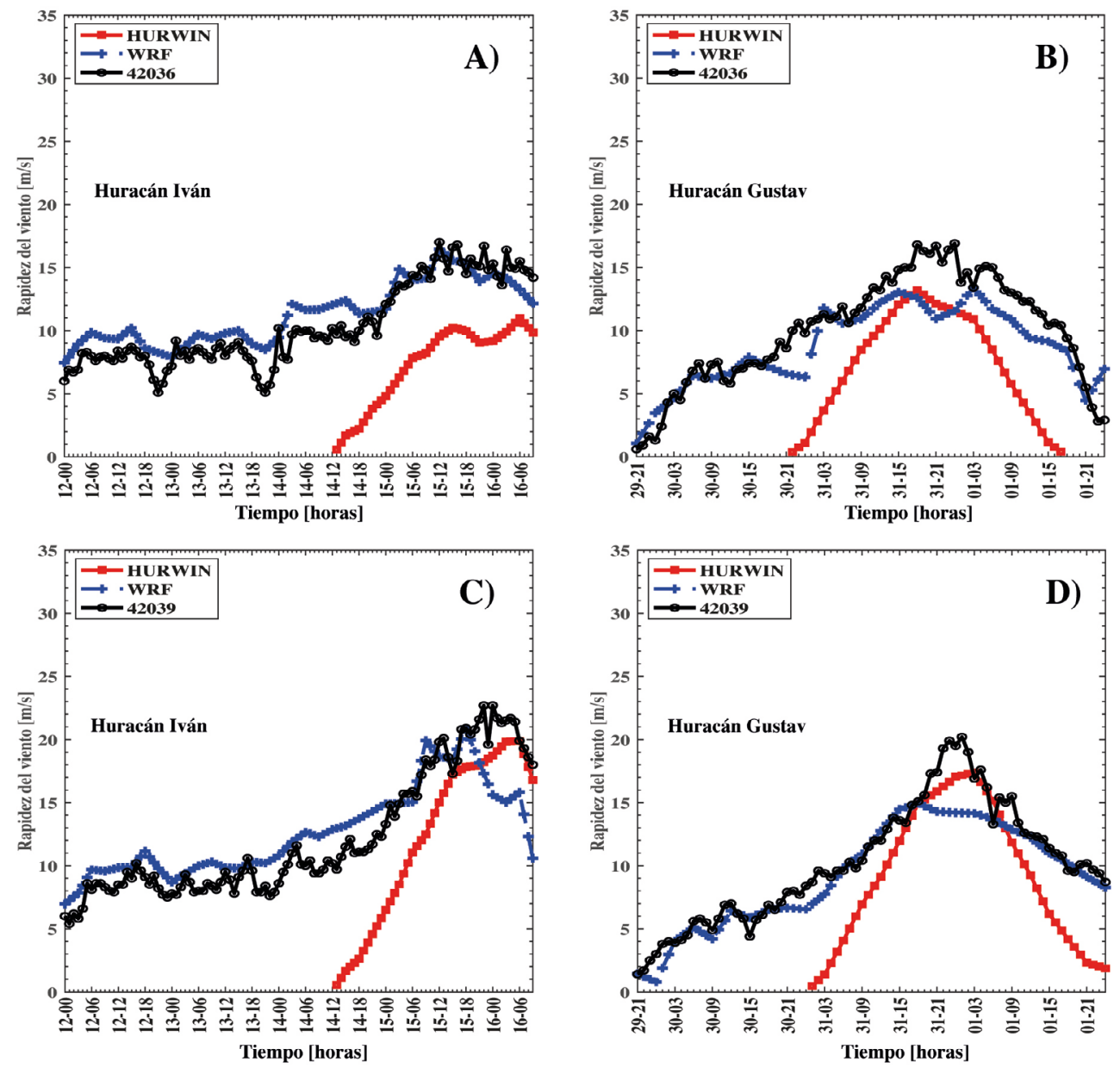

Fig. 2. Evolución temporal de la rapidez del viento (U) en boyas lejanas a la trayectoria de la tormenta. En A y C el huracán Iván 2004 para el periodo de tiempo que comprende desde el 12 de setiembre a las 00 UTC al 16 de setiembre a las 09 UTC. En B y D el huracán Gustav 2008 para el periodo de tiempo que comprende desde el 29 de setiembre a las 21 UTC al 02 de octubre a las 00 UTC. La línea negra con círculos representa las observaciones en las boyas 42036 y 42 039. La línea roja con cuadrados es la simulación de viento de HURWIN y la línea azul con cruces es la simulación de viento de WRF

Fig. 2. Time series of wind speed (U) on buoys far from the path of the storm. In A and C hurricane Ivan 2004 covering the period from September 12 at 00 UTC to September 16 at 09 UTC. In B and D hurricane Gustav 2008 covering the period from September 29 at 21 UTC to October 02 at 00 UTC. The black line with circles represents observations at buoys 42036 and 42 039. The red line with squares is the HURWIN wind speed simulation, and the blue line with crosses is the WRF wind speed simulation 

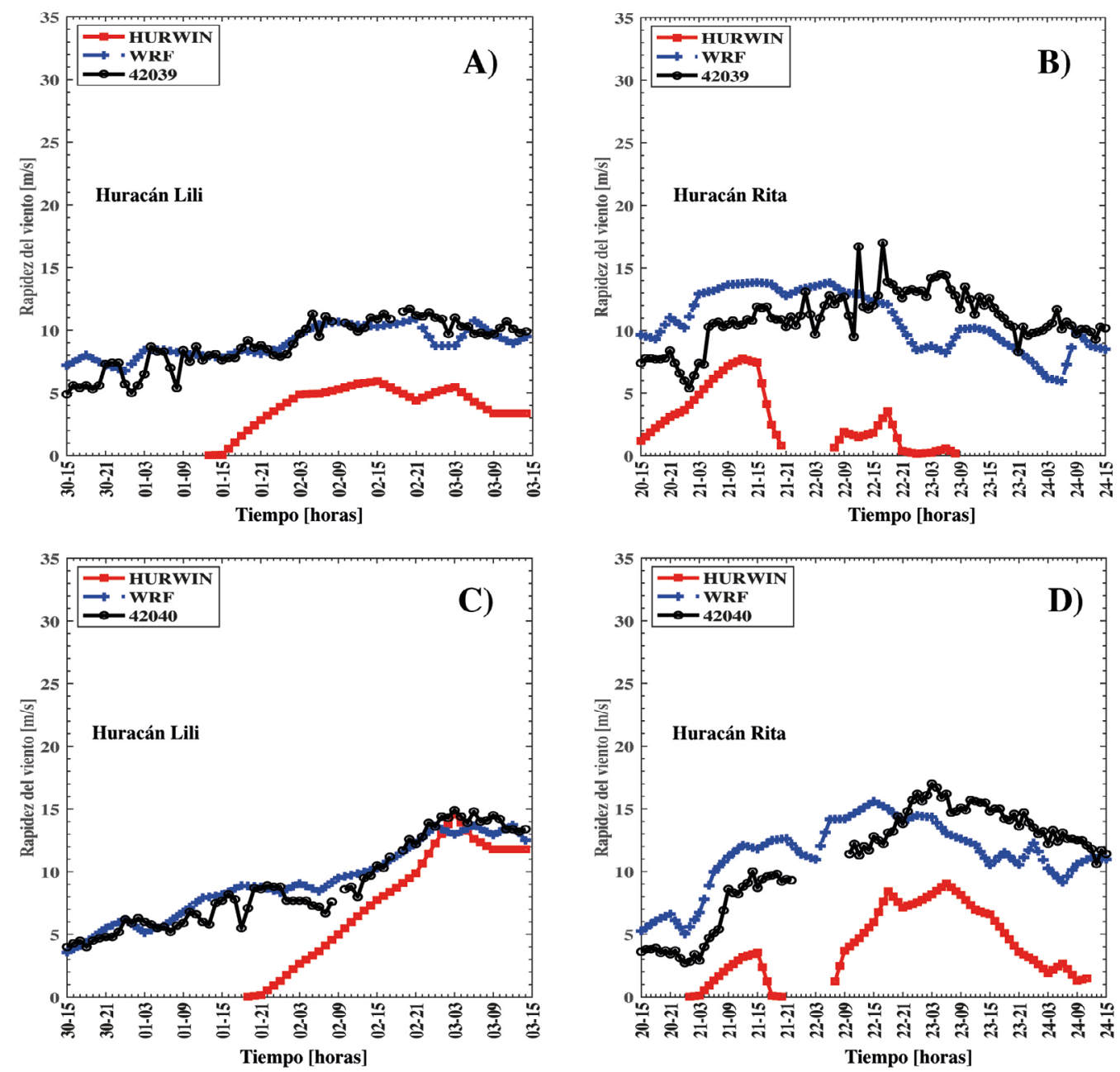

Fig. 3. Igual que en la Fig. 2 pero en A y C el huracán Lili 2002 para el periodo de tiempo que comprende desde el 12 de setiembre a las 00 UTC al 16 de setiembre a las 09 UTC. En B y D el huracán Rita para el periodo de tiempo que comprende desde el 12 de setiembre a las 15 UTC al 16 de setiembre a las 15 UTC. La línea negra con círculos representa las observaciones en las boyas 42039 y 42040

Fig. 3. Same as in Fig. 2 but in A and C hurricane Lili 2002 covering the period from September 12 at 00 UTC to September 16 at 09 UTC. In B and D hurricane Rita 2005 covering the period from September 20 at 15 UTC to September 24 at 15 UTC. The black line with circles represents observations at buoys 42039 and 42040 
Comparación de la rapidez del la boya 42003 al usar los resultados viento en boyas cercanas a la trayec- obtenidos de WRF y HURWIN. De los toria de la tormenta

En la Fig. 4 se presentan las series temporales de la rapidez del viento del huracán Lili en la boya 42 001, del Rita en la boya 42001 y del Gustav en 3 huracanes mostrados en la Fig. 4, el Lili (Fig. 4A) registró la mayor intensidad de viento, con $47.2 \mathrm{~m} \mathrm{~s}^{-1}$, siendo HURWIN (línea roja) capaz de capturar este pico de viento máximo, resultado
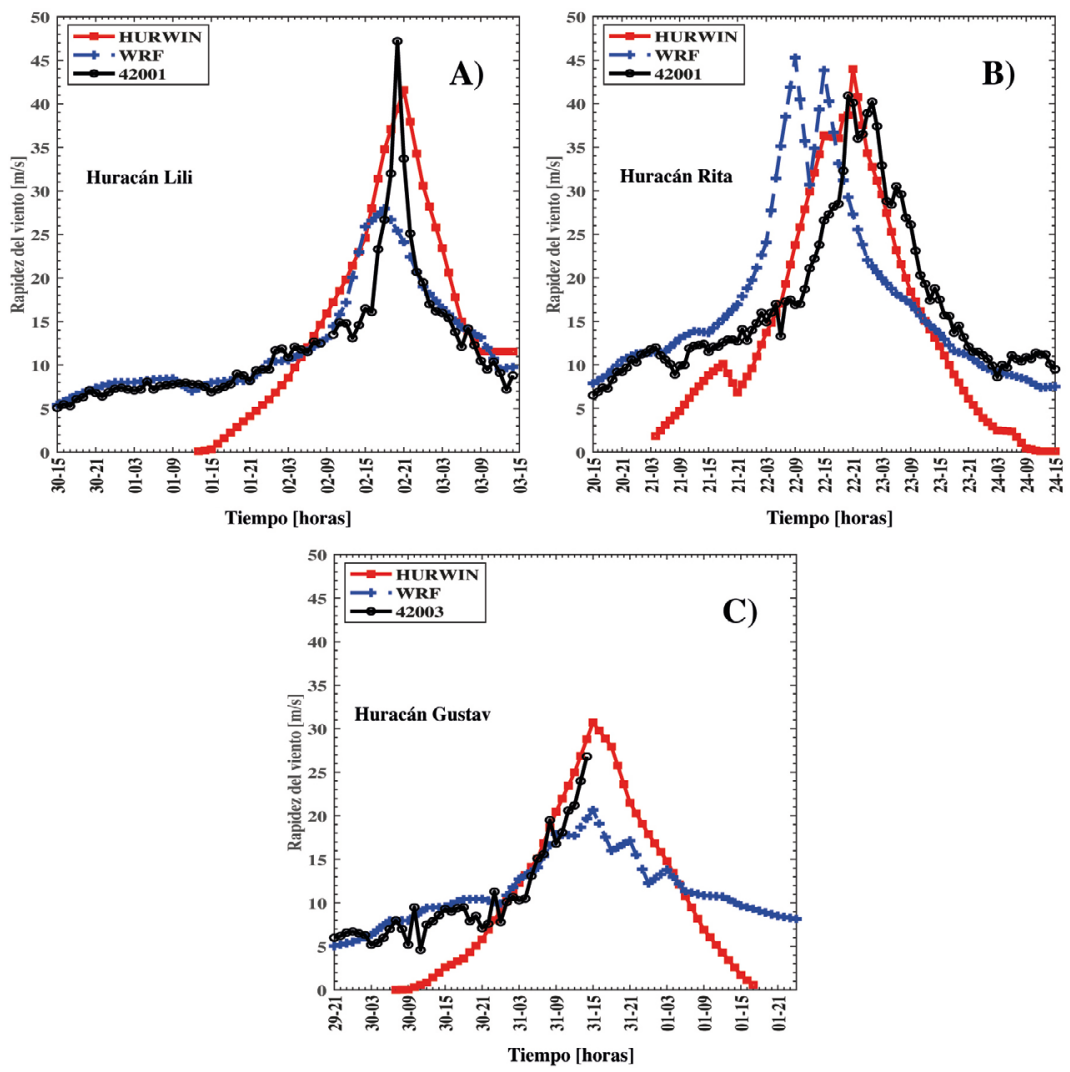

Fig. 4. Evolución temporal de la rapidez del viento (U) en boyas cercanas a la trayectoria de la tormenta. A) Huracán Lili 2002 en la boya 42 001. B) Huracán Rita en la boya 42 001. C) Huracán Gustav 2008 en la boya 42 003. La línea negra con círculos representa las observaciones en las boyas 42001 y 42 003. La línea roja y la línea azul indican lo mismo de las Fig. 2, 3

Fig. 4. Time series of wind speed (U) on buoys near the path of the storm. A) Hurricane Lili 2002 on buoy 42 001. B) Hurricane Rita 2005 on buoy 42 001. C) Hurricane Gustav 2008 on buoy 42003 . The black line with circles represents observations at buoys 42001 and 42 003. Lines red and blue indicate the same as Figures 2 and 3. 
similar para el caso del huracán Gustav (Fig. 4C), pero en la boya 42 003. Ahora, con los resultados de WRF (línea azul) ni siquiera pasa los $28 \mathrm{~m} \mathrm{~s}^{-1}$, aunque a los extremos del pico de viento máximo si es comparable con las observaciones (Figs. 4A, C) cuando todavía no es categoría huracán $\left(<30 \mathrm{~m} \mathrm{~s}^{-1}\right)$. Para el caso particular del huracán Rita (Fig. 4B), los resultados de los dos modelos de viento reproducen el máximo de viento observado en la boya, siendo HURWIN el más similar (línea roja), mientras que WRF (línea azul) presenta un desfase de unas horas antes con respecto a las observaciones y HURWIN.

\section{Evaluación de los modelos} HURWIN y WRF

Para evaluar los resultados de la rapidez del viento obtenido con los modelos HURWIN Cuadro 1. Comparación de estadísticos para los resultados de la rapidez del viento $\left(\mathrm{m} \mathrm{s}^{-1}\right)$ usando el modelo HURWIN y el modelo WRF para los huracanes Lili 2002, Iván 2004, Katrina 2005, Rita 2005 y Gustav 2008

Table 1. Statistical comparison of results for wind speed $\left(\mathrm{m} \mathrm{s}^{-1}\right)$ using the HURWIN and WRF models for hurricanes Lili 2002, Ivan 2004, Katrina 2005, Rita 2005, and Gustav 2008

\begin{tabular}{|c|c|c|c|c|c|c|c|c|}
\hline \multirow[b]{2}{*}{ Boya } & \multicolumn{4}{|c|}{ HURWIN } & \multicolumn{4}{|c|}{ WRF } \\
\hline & $\mathbf{N}$ & ME & RMSE & $\mathbf{R}$ & $\mathbf{N}$ & $\mathbf{M E}$ & RMSE & $\mathbf{R}$ \\
\hline \multicolumn{9}{|c|}{ Huracán Lili 2002} \\
\hline 42001 & 43 & 2.61 & 6.24 & 0.87 & 65 & 0.13 & 3.80 & 0.85 \\
\hline 42003 & 47 & -1.74 & 3.00 & 0.88 & 64 & 0.27 & 1.11 & 0.92 \\
\hline 42036 & 21 & -5.27 & 5.54 & -0.01 & 65 & 0.63 & 1.18 & 0.74 \\
\hline 42039 & 37 & -5.51 & 5.59 & 0.51 & 64 & -0.15 & 0.81 & 0.88 \\
\hline 42040 & 34 & -2.47 & 2.69 & 0.97 & 64 & 0.42 & 0.90 & 0.97 \\
\hline \multicolumn{9}{|c|}{ Huracán Iván 2004} \\
\hline 42003 & 58 & -5.07 & 5.40 & 0.96 & 100 & -0.42 & 3.47 & 0.86 \\
\hline 42036 & 39 & -6.02 & 6.12 & 0.88 & 100 & 1.35 & 1.83 & 0.94 \\
\hline 42039 & 40 & -4.00 & 4.66 & 0.96 & 100 & 0.73 & 2.58 & 0.87 \\
\hline 42040 & 27 & -1.84 & 5.88 & 0.97 & 91 & 3.33 & 5.09 & 0.83 \\
\hline \multicolumn{9}{|c|}{ Huracán Rita 2005} \\
\hline 42001 & 69 & -1.99 & 5.79 & 0.90 & 91 & 0.24 & 7.15 & 0.67 \\
\hline 42036 & 19 & -2.94 & 3.22 & 0.41 & 89 & 0.20 & 2.42 & 0.51 \\
\hline 42039 & 25 & -4.80 & 5.72 & 0.08 & 91 & 0.34 & 2.18 & 0.49 \\
\hline 42040 & 47 & -7.67 & 7.87 & 0.69 & 80 & 0.60 & 2.26 & 0.83 \\
\hline \multicolumn{9}{|c|}{ Huracán Gustav 2008} \\
\hline 42001 & 43 & -0.41 & 2.43 & 0.88 & 70 & 3.13 & 5.65 & 0.84 \\
\hline 42003 & 23 & -0.51 & 3.08 & 0.95 & 36 & 0.81 & 1.99 & 0.95 \\
\hline 42036 & 36 & -4.89 & 5.29 & 0.83 & 70 & -1.12 & 2.04 & 0.90 \\
\hline 42039 & 40 & -2.95 & 3.59 & 0.94 & 70 & -0.54 & 1.95 & 0.90 \\
\hline 42040 & 39 & -1.02 & 3.02 & 0.96 & 69 & -0.62 & 1.94 & 0.95 \\
\hline
\end{tabular}
y WRF para las 4 simulaciones de huracanes realizadas, en el Cuadro 1 se presentan estadísticas que permiten comparar el desempeño de los 2 modelos de viento empleados en el estudio. Es importante recalcar que el número de datos de entrada para hacer la estadística varía para cada boya y modelo de viento, solo se consideran datos de viento mayores a $2.5 \mathrm{~m} \mathrm{~s}^{-1}$ para evitar valores que introduzcan ruido en la métrica aplicada.

En términos generales, el modelo HURWIN tiende a tener valores de 
ME negativos, mientras que el modelo WRF tiende a valores positivos de ME y cercanos a la media observada. Es importante notar que los resultados de ME en las boyas 42001 y 42 003 para el modelo HURWIN son similares a los reportados por Yaghoobi Kalourazi et al. (2020), que indica que la mayoría de los modelos analíticos utilizados en su estudio subestiman la rapidez del viento. En cuanto a la varianza, el modelo HURWIN presenta ligeramente valores de RMSE mayores en comparación con el modelo WRF. En las boyas 42001 y 42 003 el modelo HURWIN presenta un coeficiente de correlación alto $(\mathrm{R}>$ $0.85)$ para los 4 huracanes simulados, mientras que el modelo WRF presenta un $\mathrm{R}>0.70$ en las boyas 42036 , 42039 y 42 040. Es importante destacar que los resultados de la rapidez del viento para el huracán Gustav al utilizar los 2 modelos de viento tienden a presentar una mejor métrica estadística en el ME, el RMSE y el R con respecto a los demás huracanes simulados. Complementario a este resultado, el modelo HURWIN tiene mejor desempeño en el ME y RMSE que los resultados reportados en Yaghoobi Kalourazi et al. (2020). Este estudio evalúa la rapidez del viento en 7 diferentes modelos paramétricos en las boyas 42001 y 42040 .
Altura del oleaje en boyas localizadas en profundidades intermedias o poco profundas

En la Fig. 5 se presenta la respuesta del campo de oleaje (con el forzamiento del viento de WRF y HURWIN) en términos del parámetro integral de la altura significante del oleaje obtenido con los resultados de WW3 (línea azul) y SWAN (línea roja) en los puntos de las boyas situadas en aguas intermedias o someras (42 036, 42039 y 42 040). De los resultados con la simulación de oleaje para el huracán Gustav (Figs. 5B, D, F) es notorio observar que se registran alturas de oleaje por arriba de los $4 \mathrm{~m}$ en las 3 boyas, siendo los resultados de altura de oleaje de WW3 y SWAN y con el modelo de viento HURWIN (véase línea azul y línea roja con cuadrados) similares a los registrados en las boyas 420036,42039 y 42040 , excepto en los 2 primeros días de simulación. Para el caso particular del huracán Gustav en la boya 42 040, Ding et al. (2020; en su figura 14) obtienen al usar con el nuevo modelo paramétrico de viento no lineal en el modelo de olas una sobreestimación de $2 \mathrm{~m}$ sobre el máximo de altura de oleaje. En el mismo estudio, pero al emplear el modelo paramétrico de Holland (1980), se sobreestima el máximo de altura de oleaje registrado en la boya 42040 en casi $5 \mathrm{~m}$. Al comparar lo obtenido por Ding et al. (2020), pareciera que los resultados de HURWIN representan mejor 

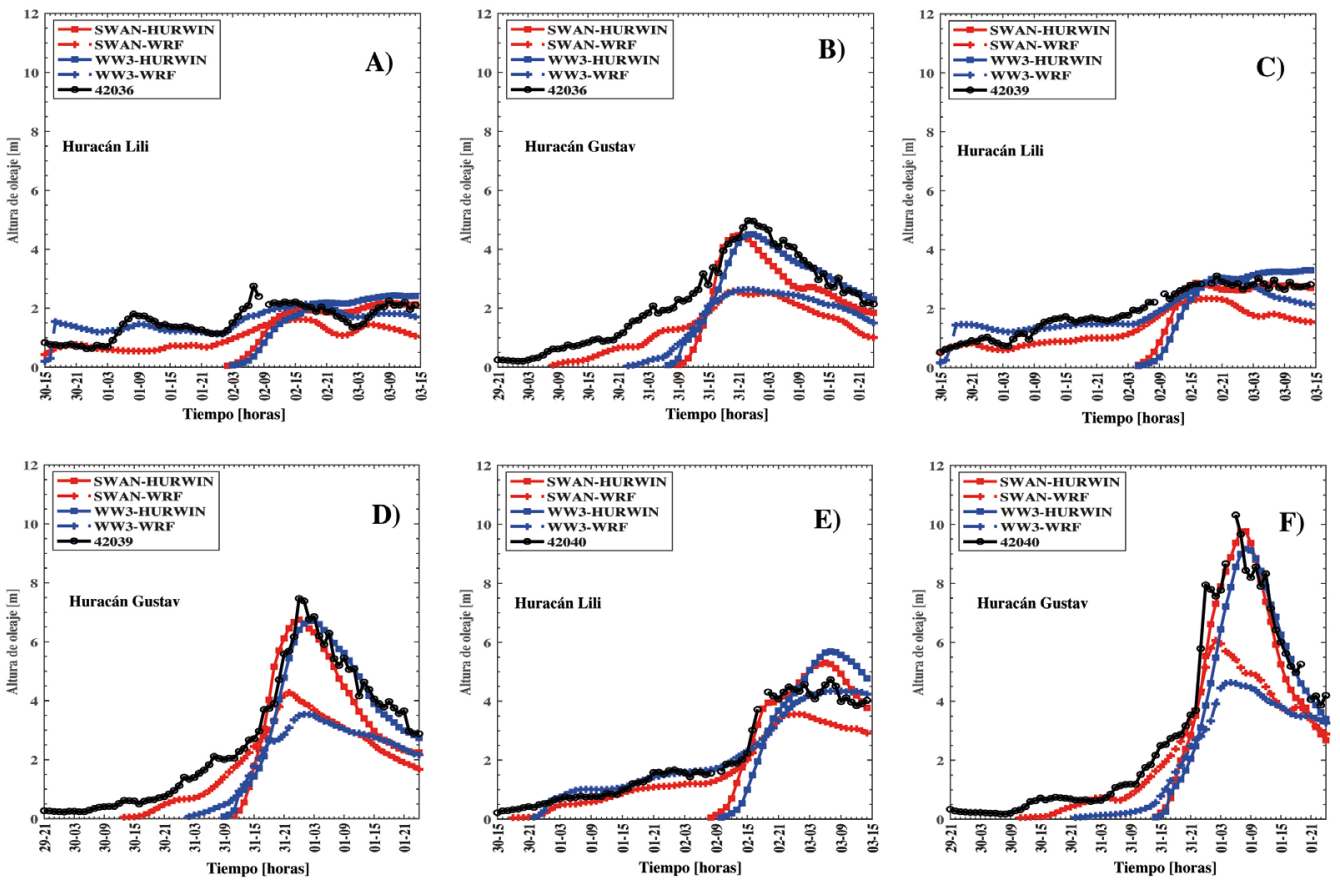

Fig. 5. Evolución temporal de la altura significante del oleaje (Hs) en boyas localizadas en aguas someras o intermedias (42 036, 42039 y 42 040). A, C y E) Huracán Lili 2002. B, D, F) Huracán Gustav 2008. La línea negra con círculos representa las observaciones en las boyas 42 036, 42039 y 42 040. La línea roja es la simulación de oleaje de SWAN con HURWIN (cuadrados) y WRF (cruces) y la línea azul es la simulación de oleaje de WW3 con HURWIN (cuadrados) y WRF (cruces)

Fig. 5. Time series of significant wave height (Hs) in buoys located in shallow or intermediate waters (42 036, 42 039, and 42 040). A, C, and E) Hurricane Lili 2002. B, D, and F) Hurricane Gustav 2008. The black line with circles represents observations at buoys 42036,42039 , and 42040 . The red line is the SWAN wave simulation with HURWIN (squares) and WRF (crosses), and the blue line is the WW3 wave simulation with HURWIN (squares) and WRF (crosses)

el pico de oleaje observado. Por otro lado, tras usar los resultados de viento del WRF (véase línea azul y línea roja con cruces) en WW3 y SWAN no llegan a reproducir el pico de oleaje máximo observado en las tres boyas.

Para el huracán Lili se observaron alturas de oleaje por arriba de los
$4 \mathrm{~m}$ solamente en la boya 42040 (Fig. $5 \mathrm{E}$ ), lo que hace los resultados de los dos modelos de oleaje forzado con HURWIN similares a las observaciones (véase línea azul y línea roja con cuadrados), excepto en los dos primeros días de simulación. En condiciones por debajo de los $4 \mathrm{~m}$ de altura de 
oleaje los dos modelos de oleaje con los resultados de WRF (véase línea azul y línea roja con cruces) tienden a ser parecidos a las observaciones (Figs. 5A, C).

En general ambos modelos de propagación de oleaje, WW3 y SWAN, reproducen lo observado en las dos boyas de profundidad intermedia (42039 y 42 040) y en la de profundidad somera (42 036), a pesar de la diferencia en su física (términos fuente) y esquemas numéricos (condición de Courant-Friedrichs-Lewy de estabilidad o inestabilidad de la simulación).

Altura del oleaje en boyas localizadas en aguas profundas

En la Fig. 6 se presentan los resultados de la simulación de oleaje de la altura significante del oleaje (huracán Lili Figs. 6A, C, E y Gustav Figs. 6B, D, F) usando WW3 y
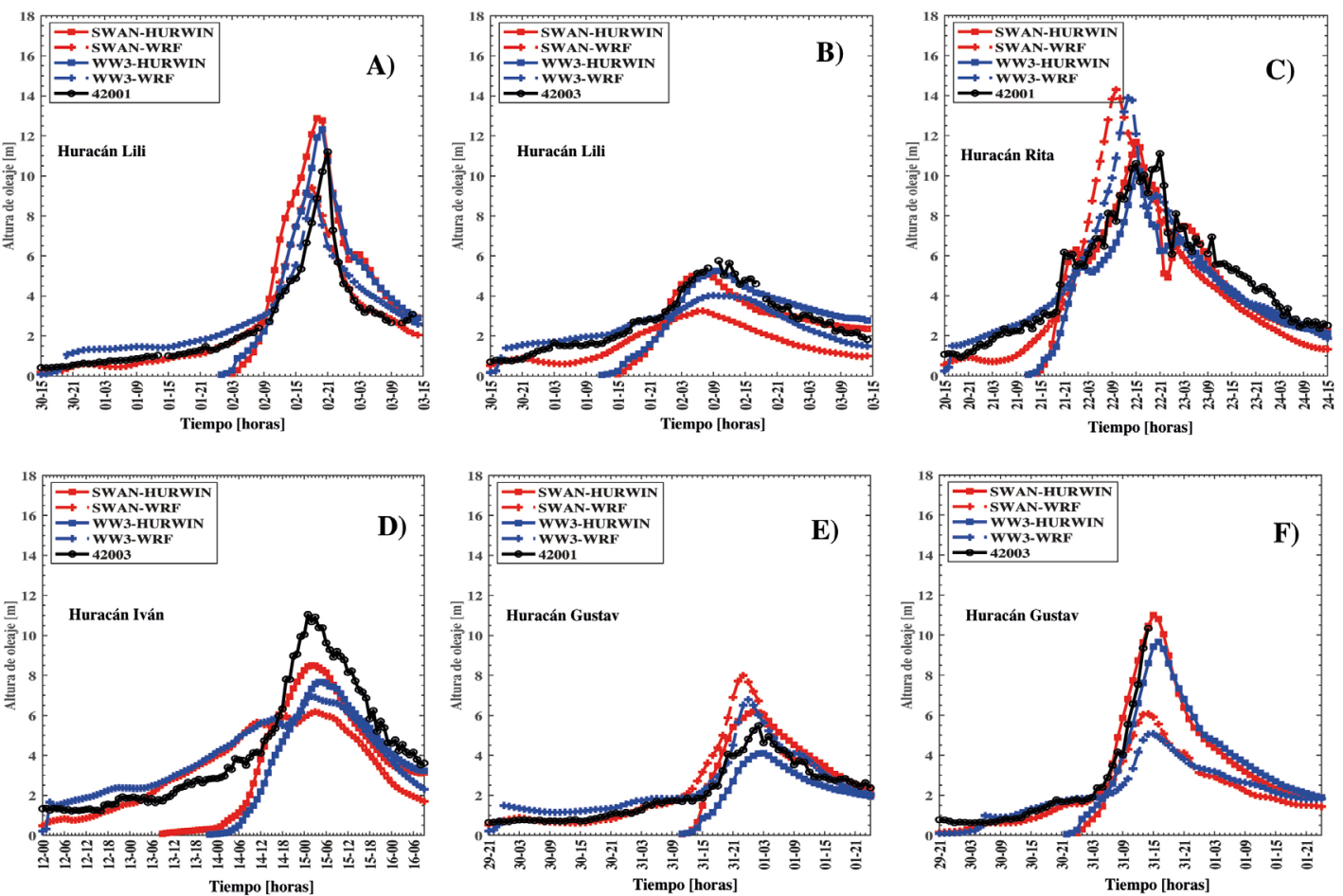

Fig. 6. Evolución temporal de la altura significante del oleaje (Hs) en boyas localizadas en aguas profundas (42 001 y 42 003). A y B) Huracán Lili 2002. C) Huracán Rita 2005. D) Huracán Iván 2004. E y F) Huracán Gustav 2008. La línea negra con círculos representa las observaciones en las boyas 42001 y 42 003. La línea roja y la línea azul indican lo mismo de la Fig. 5

Fig. 6. Time series of significant wave height (Hs) in buoys located in deep waters (42 001 and 42 003). A and B) Hurricane Lili 2002. C) Hurricane Rita 2005. D) Hurricane Ivan 2004. E and F) Hurricane Gustav 2008. The black line with circles represents observations at buoys 42001 and 42003. Lines red and blue indicate the same as Fig. 5 
SWAN con el forzamiento de los 2 modelos de viento en las 2 boyas localizadas en aguas profundas. Los registros indican que en las 2 boyas de agua profunda se presenta un pico de altura de oleaje superior o igual a los $6 \mathrm{~m}$ (excepto en la Fig. 6E), el cual es reproducido por ambos modelos de olas, siendo los resultados con HURWIN (véase línea azul y línea

Cuadro 2. Comparación de estadísticos para los resultados de la altura significante del oleaje (m) usando el modelo WAVEWATCH III y el modelo SWAN para el huracán Lili 2002 y huracán Iván 2004 Table 2. Statistical comparison of results for significant wave height $(\mathrm{m})$ using the WAVEWATCH III and SWAN models for hurricanes Lili 2002 and Ivan 2004

\begin{tabular}{|c|c|c|c|c|c|c|c|c|}
\hline \multirow[t]{2}{*}{ Boya } & \multicolumn{6}{|c|}{ Lili 2002} & \multicolumn{2}{|c|}{ Iván 2004} \\
\hline & $\mathbf{N}$ & ME & RMSE & $\mathbf{R}$ & $\mathbf{N}$ & ME & RMSE & $\mathbf{R}$ \\
\hline \multicolumn{9}{|c|}{ SWAN + HURWIN } \\
\hline 42001 & 32 & -1.69 & 2.45 & 0.90 & -- & -- & -- & -- \\
\hline 42003 & 45 & -0.45 & 0.86 & 0.81 & 54 & -1.41 & 1.62 & 0.94 \\
\hline 42036 & 32 & -0.17 & 0.62 & -0.41 & 40 & -0.95 & 1.23 & 0.83 \\
\hline 42039 & 29 & -0.21 & 0.45 & 0.80 & 40 & -2.15 & 2.56 & 0.96 \\
\hline 42040 & 26 & 0.13 & 0.57 & 0.94 & 30 & -2.25 & 2.39 & 0.99 \\
\hline \multicolumn{9}{|c|}{ SWAN + WRF } \\
\hline 42001 & 62 & 0.05 & 0.95 & 0.93 & -- & -- & -- & -- \\
\hline 42003 & 64 & -1.19 & 1.36 & 0.90 & 100 & -0.88 & 1.97 & 0.82 \\
\hline 42036 & 65 & -0.60 & 0.69 & 0.72 & 100 & -0.29 & 0.88 & 0.90 \\
\hline 42039 & 64 & -0.65 & 0.71 & 0.93 & 100 & -0.80 & 2.32 & 0.81 \\
\hline 42040 & 26 & -0.86 & 0.93 & 0.95 & 89 & 0.44 & 1.67 & 0.88 \\
\hline \multicolumn{9}{|c|}{ WAVEWATCH III + HURWIN } \\
\hline 42001 & 32 & 1.21 & 1.75 & 0.93 & -- & -- & -- & -- \\
\hline 42003 & 45 & -0.13 & 0.74 & 0.81 & 54 & -1.86 & 2.13 & 0.91 \\
\hline 42036 & 32 & -0.06 & 0.80 & -0.53 & 40 & -1.40 & 1.45 & 0.96 \\
\hline 42039 & 29 & -0.07 & 0.72 & 0.79 & 40 & -2.95 & 3.05 & 0.98 \\
\hline 42040 & 26 & 0.06 & 1.14 & 0.87 & 30 & -3.67 & 3.78 & 0.99 \\
\hline \multicolumn{9}{|c|}{ WAVEWATCH III + WRF } \\
\hline 42001 & 62 & 0.39 & 0.94 & 0.94 & -- & -- & -- & -- \\
\hline 42003 & 64 & -0.31 & 0.67 & 0.95 & 100 & -0.34 & 1.61 & 0.90 \\
\hline 42036 & 65 & -0.04 & 0.31 & 0.78 & 100 & -0.06 & 0.66 & 0.96 \\
\hline 42039 & 64 & -0.12 & 0.33 & 0.91 & 100 & -0.50 & 1.84 & 0.93 \\
\hline 42040 & 26 & -0.23 & 0.57 & 0.81 & 89 & -0.43 & 1.54 & 0.91 \\
\hline
\end{tabular}

roja con cuadrados) los más parecidos a las observaciones. En los extremos de este máximo de altura de oleaje, la simulación con ambos modelos de oleaje y los resultados de WRF (véase línea azul y línea roja con cruces) son comparables con los datos medidos por las boyas 42001 y 42003 .

En general ambos modelos numéricos de oleaje, WW3 y SWAN, logran reproducir condiciones extremas de oleaje, a pesar de las diferencias en los esquemas numéricos de propagación y en las parametrizaciones físicas implementadas.

Ev a l u a c i ó n de los modelos WAVEWATCH Y SWAN Los resultados estadísticos para los modelos WW3 y SWAN en los huracanes Lili e Iván se presentan en el Cuadro 2 y para los huracanes Rita y Gustav se presentan en el Cuadro 3. Es importante destacar que el número de datos de entrada para hacer la estadística varía para cada boya y modelo de oleaje, solo se consideran alturas de oleaje por arriba de $0.1 \mathrm{~m}$. 
Cuadro 3. Igual que en el Cuadro 2 pero para el huracán Rita 2005 y huracán Gustav 2008

Table 3. Same as Table 2 but for hurricanes Rita 2005 and Gustav 2008

\begin{tabular}{|c|c|c|c|c|c|c|c|c|}
\hline \multirow[t]{2}{*}{ Boya } & \multicolumn{4}{|c|}{ Rita 2005} & \multicolumn{4}{|c|}{ Gustav 2008} \\
\hline & $\mathbf{N}$ & ME & RMSE & $\mathbf{R}$ & $\mathbf{N}$ & ME & RMSE & $\mathbf{R}$ \\
\hline \multicolumn{9}{|c|}{ SWAN + HURWIN } \\
\hline 42001 & 72 & -0.52 & 1.16 & 0.93 & 35 & 0.59 & 0.83 & 0.96 \\
\hline 42003 & -- & -- & -- & -- & 14 & 0.00 & 1.08 & 0.97 \\
\hline 42036 & 85 & -0.72 & 0.90 & 0.61 & 38 & -0.70 & 0.89 & 0.85 \\
\hline 42039 & 74 & -1.09 & 1.29 & 0.54 & 37 & -0.64 & 0.98 & 0.93 \\
\hline 42040 & 58 & -2.04 & 2.14 & 0.85 & 31 & -0.72 & 1.09 & 0.97 \\
\hline \multicolumn{9}{|c|}{ SWAN + WRF } \\
\hline 42001 & 90 & -0.44 & 1.94 & 0.88 & 70 & 0.39 & 1.01 & 0.92 \\
\hline 42003 & -- & -- & -- & -- & 36 & -0.57 & 1.06 & 0.98 \\
\hline 42036 & 90 & -0.44 & 1.94 & 0.88 & 38 & -1.52 & 1.59 & 0.95 \\
\hline 42039 & 89 & -0.94 & 1.03 & 0.79 & 37 & -1.66 & 1.87 & 0.85 \\
\hline 42040 & 91 & -1.09 & 1.30 & 0.81 & 31 & -1.91 & 2.32 & 0.91 \\
\hline \multicolumn{9}{|c|}{ WAVEWATCH III + HURWIN } \\
\hline 42001 & 72 & -1.13 & 1.46 & 0.92 & 35 & -0.81 & 0.89 & 0.93 \\
\hline 42003 & -- & -- & -- & -- & 14 & -0.90 & 0.99 & 0.99 \\
\hline 42036 & 85 & -0.60 & 0.76 & 0.70 & 38 & -0.34 & 0.61 & 0.85 \\
\hline 42039 & 74 & -1.00 & 1.19 & 0.63 & 37 & -0.39 & 0.72 & 0.95 \\
\hline 42040 & 58 & -2.00 & 2.06 & 0.93 & 31 & 0.88 & 1.49 & 0.89 \\
\hline \multicolumn{9}{|c|}{ WAVEWATCH III + WRF } \\
\hline 42001 & 90 & -0.01 & 1.22 & 0.92 & 70 & 0.35 & 0.57 & 0.96 \\
\hline 42003 & -- & -- & -- & -- & 14 & -1.83 & 2.50 & 0.99 \\
\hline 42036 & 89 & -0.21 & 0.63 & 0.57 & 38 & -1.30 & 1.41 & 0.89 \\
\hline 42039 & 91 & -0.25 & 0.80 & 0.78 & 37 & -1.86 & 2.06 & 0.91 \\
\hline 42040 & 80 & -0.51 & 0.93 & 0.95 & 31 & -2.57 & 2.99 & 0.85 \\
\hline
\end{tabular}

las boyas 42001 y 42003. Es importante destacar que los resultados de altura de oleaje para el huracán Gustav con los 2 modelos de oleaje tienden a presentar mejores métricas estadísticas, que con los resultados de la simulación de oleaje para los huracanes Lili, Iván y Rita.

\section{DISCUSIÓN}

Las series temporales de rapidez del viento con los resultados del modelo paramétrico HURWIN mostraron ser similares, en particular, al capturar el máximo observado cuando la boya se encuentra cerca de la trayectoria de la tormenta, como es el caso de las boyas 42001 (Lili
En general la métrica aplicada en los 4 huracanes simulados nos indica que los modelos de oleaje, WW3 y SWAN presentan valores de ME negativos en la mayoría de las boyas evaluadas. Por otro lado, el modelo WW3 presenta una mayor precisión (RMSE) en la mayoría de las boyas si se compara con el modelo SWAN. En cuanto al patrón observado, WW3 presenta ligeramente mejor desempeño en el estadístico R que el modelo SWAN en y Rita) y 42003 (Gustav) mostradas en la Fig. 4. Esto es consistente con lo que indica Ruiz-Salcines (2019) de que el campo de viento de los modelos paramétricos está en función de la distancia del ojo del huracán. Lejos del centro de la tormenta (a distancias mayores a $400 \mathrm{~km}$ ) el modelo HURWIN no es consistente con los datos registrados de intensidad en las boyas, debido a que el campo de viento más allá del radio de vientos máximo decae 
exponencialmente hasta cero a grandes distancias. La deficiencia de este modelo paramétrico está en que no representan el flujo geostrófico ambiental como lo menciona Lizano (2006).

Si bien el uso de un modelo complejo como WRF puede representar la física de los procesos atmosféricos, no garantiza que tenga la habilidad de representar el máximo de viento de un huracán, tiende a subestimar el pico de intensidad de viento en los casos de Lili (Fig. 4A) y Gustav (Fig. 4C) y a sobreestimar el máximo de viento en el caso de Rita (Fig. 4B). Un argumento válido es la resolución espacial escogida, que conlleva a una pobre representación de la estructura interna, a los procesos de intensificación en el núcleo, $\mathrm{y}$, por lo tanto, a una subestimación de la intensidad del campo de vientos. En el caso de Rita de la Fig. $4 \mathrm{~B}$, asociamos al hecho de que la trayectoria del huracán no es bien representada, como si lo es en HURWIN, que se basa en parámetros del huracán para seguir la mejor trayectoria. El modelo WRF tiende a ser comparable con las boyas que se ubican lejos del centro de la tormenta (42 036, 42039 y 42 040) donde la influencia de su ojo es casi nula (en la mayoría de los casos simulados). Esto es así, porque el modelo WRF tiene datos de viento a esas distancias generadas por las condiciones de frontera de NCEP/FNL.

En cuanto a la respuesta de los dos modelos espectrales en la altura de oleaje, los resultados con el modelo HURWIN mostraron capturar el máximo observado en las boyas $42001 \mathrm{y}$ 42003 para los huracanes Lili, Rita y Gustav. Por otro lado, los resultados de altura de oleaje con el modelo WRF presenta en la mayoría de las boyas una respuesta comparable con las observaciones lejos de la influencia de los vientos intensos, a diferencia de otros estudios, donde indican una mejor representación de la altura significante del oleaje en WW3 que en SWAN (Ortiz-Royero \& Mercado-Irizarry, 2008). Es importante mencionar que la estructura e intensidad de un huracán están relacionados con la resolución espacial y temporal escogido, en este caso $\sim 10 \mathrm{~km}$ y 3 horas. Además, la trayectoria de la tormenta juega un papel importante con respecto a la distancia con las boyas. Estos factores modifican la calidad de los campos de viento que se obtiene, y por ende en la estructura del campo de oleaje.

El desempeño estadístico de los modelos de viento sugiere que el modelo HURWIN tiende a subestimar la intensidad del viento (valores negativos de ME), resultado independiente de la distancia a la que se encuentre la boya con el centro de la tormenta. Yaghoobi Kalourazi et al. (2020) menciona que la subestimación del viento en los modelos analizados de su trabajo se puede mejorar, al calibrar los parámetros que controlan la forma del huracán. Por otro lado, Young (2017) 
indica que para grandes distancias con respecto al centro de la tormenta el viento es subestimado en el modelo paramétrico de Holland (1980). En efecto, el argumento de Young (2017) pareciera justificar los valores negativos en la métrica obtenida. Para el modelo WRF, este tiende ligeramente a sobreestimar la magnitud del viento (valores de ME positivos). Estos dos resultados tienen implicaciones importantes en los flujos de energía y momento que se transfieren a un modelo de oleaje.

Por otro lado, los 2 modelos de oleaje tienden a subestimar la altura de oleaje observada según el estadístico ME para los 4 huracanes simulados. Dos causas que argumentamos, la escala espacial de los campos de viento y la formulación física establecida en el modelo espectral. Con respecto a este último, por ejemplo, en WW3, se establece un coeficiente de arrastre a un valor predeterminado en el término de crecimiento del suministro de energía de Tolman \& Chalikov (1996), por ende, se da una subestimación en el área de mayor energía del huracán (Montoya et al. 2013). Los resultados de la serie temporal de la altura de oleaje para el huracán Gustav tienden a parecerse a las observaciones, por ende, el buen desempeño obtenido en los tres estadísticos evaluados. Este resultado en particular y no en los otros 3 huracanes simulados, pareciera confirmar lo que indica Ruiz-Salcines et al. (2019) sobre el desempeño que se puede obtener para ciertos eventos de huracanes.

\section{CONCLUSIONES}

La respuesta de 2 modelos espectrales de olas fue evaluada a través del parámetro integral de la altura del oleaje usando 2 fuentes diferentes de viento: un modelo paramétrico y otro modelo dinámico. El modelo HURWIN representa los máximos de energía del viento registrados en las boyas cercanas a la trayectoria del huracán. Las condiciones ambientales de viento son bien representadas por el modelo WRF.

La altura del oleaje de los dos modelos de olas es comparable con las observaciones cuando el forzamiento del modelo WRF es similar a los datos de rapidez de viento de las boyas. Cuando el campo de viento de HURWIN es menor que las observaciones de rapidez de viento, la respuesta de los 2 modelos de oleaje pareciera compensar esta subestimación del viento, es decir, la altura del oleaje es ligeramente menor o igual a las observaciones en las boyas. Los dos modelos de oleaje capturan los máximos de altura de oleaje cuando el modelo HURWIN logra representar los máximos de viento.

Los resultados sugieren unir los campos de viento del modelo paramétrico HURWIN y el modelo WRF para 
una mejor representación de la estructura interna y externa de un huracán.

Como temas futuros a abordar:1) evaluar la respuesta del campo de oleaje (en términos de los parámetros integrales del espectro de olas no considerados en el estudio) bajo el campo de viento combinado con más casos de huracanes y 2) evaluar las parametrizaciones físicas más recientes en el término de suministro de energía y disipación incluidas en los modelos de oleaje para representar las condiciones de viento extremo usando el campo de viento combinado.

\section{AGRADECIMIENTOS}

Al Colaboratorio Nacional de Computación Avanzada (CNCA) del Centro Nacional de Alta Tecnología (CENAT) por las facilidades en el uso de la plataforma computacional Kabré. A la Vicerrectoría de Investigación por su apoyo al proyecto $\mathrm{N} .^{\circ}$ 805-A6-103 adscrito al Centro de Investigaciones Geofísicas (CIGEFI) de la Universidad de Costa Rica. A los evaluadores anónimos por sus comentarios que aportaron para elaborar un mejor artículo.

\section{BIBLIOGRAFÍA}

Amante, C. \& Eakins, B. (2009). ETOPO1 1 Arc-Minute Global Relief Model: Procedures, Data Sources and Analysis. EE. UU.: National Geophysical Data Center.
Booij, N., Ris, R. C. \& Holthuijsen, L. H. (1999). A third-generation wave model for coastal regions: 1. Model description and validation. J. Geophys. Res. Oceans (1978-2012), 104, 7649-7666. https://doi.org/10.1029/98JC02622

Braun, S. A. \& Tao, W.-K. (2000). Sensitivity of high-resolution simulations of hurricane Bob (1991) to planetary boundary layer parameterizations. Mon. Weather Rev., 128(12), 3941-3961. https://doi.org/10.1175/1520-0493(2000)129<3941:$\mathrm{SOHRSO}>2.0 . \mathrm{CO} ; 2$

Chen, W. B., Chen, H., Hsiao, S. C., Chang, C. C. \& Lin, L. Y. (2019). Wind forcing effect on hindcasting of typhoon-driven extreme waves. Ocean Eng., 188, 106260. https:// doi.org/10.1016/j.oceaneng.2019.106260

Collins, J. I. \& Viehnaman, J. (1971). A simplified empirical model for hurricane wind fields. EE. UU.: Offshore Technology Conference. https://doi. org/10.4043/1346-MS

Davis, C., Wang, W., Chen, S. S., Chen, Y., Corbosiero, K., DeMaría, M., ... \& Michalakes, J. (2008). Prediction of landfalling hurricanes with the advanced hurricane WRF Model. Mon. Wea. Rev., 136(6), 1990-2005. https://doi. org/10.1175/2007MWR2085.1

Ding, Y., Ding, T., Rusdin, A., Zhang, Y. \& Jia, Y. (2020). Simulation and prediction of storm surges and waves using a fully integrated process model and a parametric cyclonic wind model. $J$. Geophys. Res. Oceans, 125. https://doi. org/10.1029/2019JC015793

Emanuel, K. \& Rotunno, R. (2011). Self-Stratification of Tropical Cyclone Outflow. Part I: Implications for Storm Structure. J. Atmos. Sci., 68, 2236-2249. https://doi. org/10.1175/JAS-D-10-05024.1 
Holland, G. J. (1980). An analytic model of the wind and pressure profiles in hurricanes. Mon. Wea Rev., 108(8), 1212-1218. https://doi.org/10.1175/1520-0493(198 0) $108<1212$ :AAMOTW $>2.0 . \mathrm{CO} ; 2$

Holland, G. J., Belanger, J. I. \& Fritz, A. (2010). A revised model for radial profiles of hurricane winds. Mon. Wea. Rev., 138(12), 4393-4401. https://doi. org/10.1175/2010MWR3317.1

Holthuijsen, L. H. (2010). Waves in oceanic and coastal waters. EE. UU.: Cambridge University Press. https://doi. org/10.1017/CBO9780511618536

Hu, K., Chen, Q. \& Kimball, S. K. (2012). Consistency in hurricane surface wind forecasting: an improved parametric model. Nat. Hazards, 61, 1029-1050. https://doi.org/10.1007/ s11069-011-9960-z

Komen, G. J., Cavaleri, L., Donelan, M., Hasselmann, K., Hasselmann, S. \& Janssen, P. A. E. M. (1994). Dynamics and Modelling of Ocean Waves. EE. UU.: Cambridge University Press. https:// doi.org/10.1017/CBO9780511628955

Jelesnianski, C. (1974). Splash (special program to list amplitudes of surges from hurricanes). Part II: General tracks and variants storm conditions. EE. UU.: NOAA, Tech. Mem., NWS TDL-52.

Landsea, C. W. \& Franklin, J. L. (2013). Atlantic hurricane database uncertainty and presentation of a new database format. Mon. Wea. Rev., 141(10), 3576-3592. https://doi.org/10.1175/ MWR-D-12-00254.1

Lizano, O. G. (1990). Un modelo de viento ajustado a un modelo de generación de olas para el pronóstico de oleaje durante huracanes. Rev. Geofisica, 33, 76-103.

Lizano, O. G. \& Moya, R. (1990). Simulación de oleaje durante el huracán Joan (1988) a su paso por el mar Caribe de Costa Rica. Rev. Geofisica, 33, 105-126.
Lizano, O. G., Ocampo, F. J., Gamboa, A., Fernando, L., Vega, R. \& Puig, J. M. (2001). Evaluación de modelos numéricos de tercera generación para el pronóstico del oleaje en Centroamérica y México. Top. Meteor. Oceanogr., 8(1), 40-49.

Lizano, O. G. (2006). Simulación de oleaje de huracán usando modelos de viento paramétricos en un modelo de olas de tercera generación. Bol. Cientif. CIOH, 24, 36-47. https://doi. org/10.26640/22159045.147

Lizano, O. G. \& Mora-Escalante, R. (2019). Simulación de las condiciones oceanográficas de los huracanes que han pasado más cerca de Costa Rica. Rev. Torno Prev. CNE, (22), 21-31.

Montoya, R. D., Arias, A. O., Royero, J. O. \& Ocampo-Torres, F. J. (2013). A wave parameters and directional spectrum analysis for extreme winds. Ocean Eng., 67, 100-118. https://doi.org/10.1016/j.oceaneng.2013.04.016

Moon, I. J., Ginis, I., Hara, T., Tolman, H. L., Wright, C. \& Walsh, E. J. (2003). Numerical simulation of sea surface directional wave spectra under hurricane wind forcing. J. Phys. Oceanogr., 33(8), 1680-1706. https://doi. org/10.1175/2410.1

Mooney, P., Gill, D., Mulligan, F. \& Bruyère, C. (2016). Hurricane simulation using diferent representations of atmosphere-ocean interaction: the case of Irene (2011). Atmo. Sc. Lett., 17(Issue 7), 415-421. https://doi.org/10.1002/ asl.673

Olfateh, M., Callaghan, D. P, Nielsen, P. \& Baldock, T. E. (2017). Tropical cyclone wind field asymmetry-development and evaluation of a new parametric model. J. Geophys. Res. Oceans, 122(1), 458-469. https://doi. org/10.1002/2016JC012237 
Ortiz-Royero, J. C., Martínez, F. L, Estrada, E. D. \& Arango, L. F. (2008). Estudio del oleaje generado por el huracán Joan en la costa caribe colombiana en 1988 , incluyendo a la Isla Andrés. Rev. Colomb. Fis., 40(2), 444-446.

Ortiz-Royero, J. C. \& Mercado-Irizarry, A. (2008). An Intercomparison of Swan and Wavewatch III Models with Data from NDBC-NOAA Buoys at Oceanic Scales. Coast. Eng. J., 50(1), 47-73. https://doi.org/10.1142/ S0578563408001739

Rogers, R., Chen, S., Tenerelli, J. \& Willoughby, H. (2003). A numerical study of the impact of vertical shear on the distribution of rainfall in hurricane bonnie (1998). Mon. Wea Rev., 131(8), 15771599. https://doi.org/10.1175//2546.1

Ruiz-Salcines, P., Salles, P., Robles-Díaz, L., Díaz-Hernández, G., Torres-Freyermuth, A. \& Appendini, C. M. (2019). On the Use of Parametric Wind Models for Wind Wave Modeling under Tropical Cyclones. Water, 11(10), 2044. https:// doi.org/10.3390/w11102044

Schiller, A. \& Brassington, G. B. (2011). Operational Oceanography in the 21 st century. In A. Schiller \& G. B. Brassington (Eds.), Ocean Forecasting. System: Product Evaluation and Skill (pp. 601631), Netherlands: Springer.

Shao, Z., Liang, B., Li, H., Wu, G. \& Wu, Z. (2018). Blended wind fields for wave modeling of tropical cyclones in the South China Sea and East China Sea. Appl. Ocean Res., 71, 20-33. https:// doi.org/10.1016/j.apor.2017.11.012

Skamarock, W. C., Klemp, J., Dudhia, J., Gill, D., Barker, D., Duda, M., ... \& Powers, J. (2008). A description of the advanced research wrf version 3 . EE. UU.: NCAR/TN-475+STR.
Tolman, H. L. (2014). User manual and system documentation of WAVEWATCH III vं version 4.18. EE.UU.: NOAA/ NWS/NCEP/MMAB Tech. Note 316.

Tolman, H. L. \& Chalikov, D. (1996). Source terms in a third-generation wind-wave model. J. Phys. Oceanogr., 26(11), 2497-2518. https://doi.org/10.1175/1520-0485(1996)026<2497:STIATG $>2.0 . \mathrm{CO} ; 2$

Vickery, P. J. \& Wadhera, D. (2008). Statistical models of holland pressure profile parameter and radius to maximum winds of hurricanes from flight-level pressure and $\mathrm{H}^{*}$ Wind Data. J. Appl. Meteorol. Climatol., 47(10), 2497-2517. https:// doi.org/10.1175/2008JAMC1837.1

Willoughby, H. E. \& Rahn, M. E. (2004). Parametric representation of the primary hurricane vortex. Part I: Observations and evaluation of the Holland (1980) Model. Mon. Wea Rev., 132(12), 3033-3048. https://doi.org/10.1175/MWR2831.1

Yaghoobi Kalourazi, M., Mostafa Siadatmousavi, S., Yeganeh-Bakhtiary, A. \& Jose, F. (2020). Simulating tropical storms in the Gulf of Mexico using analytical models. Oceanologia, 62(2), 173-189. https:// doi.org/10.1016/j.oceano.2019.11.001

Young, I. R. (2017). A Review of Parametric Descriptions of Tropical Cyclone Wind-Wave Generation. Atmosphere, 8(10), 194. https://doi.org/10.3390/ atmos 8100194 
